Scrinic Olesea, Ibadula Seila, Circo E.

\title{
Graves Disease And Down Sindrome : Clinical Gase
}

Department of Endocrinology, Faculty of Medicine, "Ovidius" University of Constanta

\begin{abstract}
Introduction: Pacients with Down's syndrome present an increase revalence of autoimune endocrine disorders. We communicate the case of 14 years and 6 months old pacient known with Down syndrome admitted in Endocrinology department with suspicion of hyperthyroidism, the diagnosis being confirmed by hormonal dosage. The particularity of the case consists in: symptomatology onset during puberty, clinical evolution with mild symptoms, without ocular involvement, morphological and functional remission obtained relatively soon after the initiation of antithyroid therapy, lack of posttherapy side effects, favorabile evolution under the "block and replace" therapy.
\end{abstract}

Keywords: Down syndrome, Graves disease, puberty.

\section{Eduard Circo}

Fagetului str. 163 bis, 900654, Constanta, Romania, phone: + 40241512251; email: eduard_circo@yahoo.com;

\section{Introduction}

Down syndrome (DS) represents the most frequent genetic anomaly with an incidence of approx. 1 in 750-800 births.[1] Thyroid dysfunction is common in people with DS. Autoimmune endocrine and non-endocrine disorders (hypothyroidism, celiac disease, diabetes type 1) show a higher prevalence in people with Down's syndrome compared to the general population.[2] During childhood and adolescence chronic autoimmune thyroiditis is among the most frequent endocrine disorders (predominantly after age of 8 ) while hyperthyroidism is more rare. $[3,4,5]$

\section{Case presentation:}

A patient of 14 years and 6 months old, with Down's syndrome is hospitalized for weight loss, approx.6 kg in 1.5 months, sweating, psychomotor 
agitation, sweating, irritability, tremor of the extremities, palpitations, spaniomenorrhea.

Personal physiological history: the first born of the family, born at 8 months pregnancy, with regular prenatal checkups, normal birth, birth weight -3150 gr, height - $51 \mathrm{~cm}$; neonatal jaundice approx. 2 weeks that required phototherapy. Prevention of rickets and vaccination schedule according to the national protocol. Menarche at 10.5 years, regular menses, of approximately 4-5 days.

Personal pathological history: diagnosed at age of 1.5 months with Down's syndrome. Delayed psychomotor development: head control at 1 year, sitting at 1 year 4 months, sustained standing at 1 year 6 months.

Family medical history: father - dead at age of 41 of laryngeal cancer, paternal grandmother thyroidectomy for multi-nodular goiter.

Admission physical examination: conscious, partly cooperative, delayed language development, afebrile, height $-143.5 \mathrm{~cm}$, weight-53 kg, BMI $25.7 \mathrm{~kg} / \mathrm{m}^{2}$; BP- 120/70 mmHg, HR- 120 b / min, regularly. Clammy, warm skin, facial and chest acne eruption, laterocervical micro-adenopathy, hypotonic tissue, typical Down's syndrome facies, orizontal strabismus, known since the age 6 months, partially corrected by optical methods. The pacient wears corrections eyeglass for medium myopia since the age of 11.Grade II goiter (World Health Organisation -WHO), mobile, painless on palpation or spontaneously, homogeneous, elastic, with minimal vascular thrill (objective); without bowel disorders (mother's affirmation). Ocular motility examination without eye movement asinergy.

Following the medical history and physical examination the clinical diagnosis is established as hyperthyroidism.

For more accurate diagnosis the following laboratory investigations were made:

Hormonal dosage: TSH - 0.005 microUI/L; FT4-> $100 \mathrm{pmol} / 1$; FT3- $9.2 \mathrm{nmol} / \mathrm{L}$; TPOAb $=501$ IU / ml (NL: <34), TGAb = 74 IU / ml (NL <115), $\mathrm{TRAb}>40 \mathrm{IU} / \mathrm{L}(\mathrm{NL}<1.75) ; \mathrm{FSH}-17,8 \mathrm{mUI} / \mathrm{ml}$ (1.7-21.5) ;LH - 47, 8 mUI/ml (1-95.6); Estradiol $257 \mathrm{pmol} / 1$ (46.0-607- follicular phase) ; Progesteron$0,45 \mathrm{nmol} / 1$ (5.3-86- luteal phase);

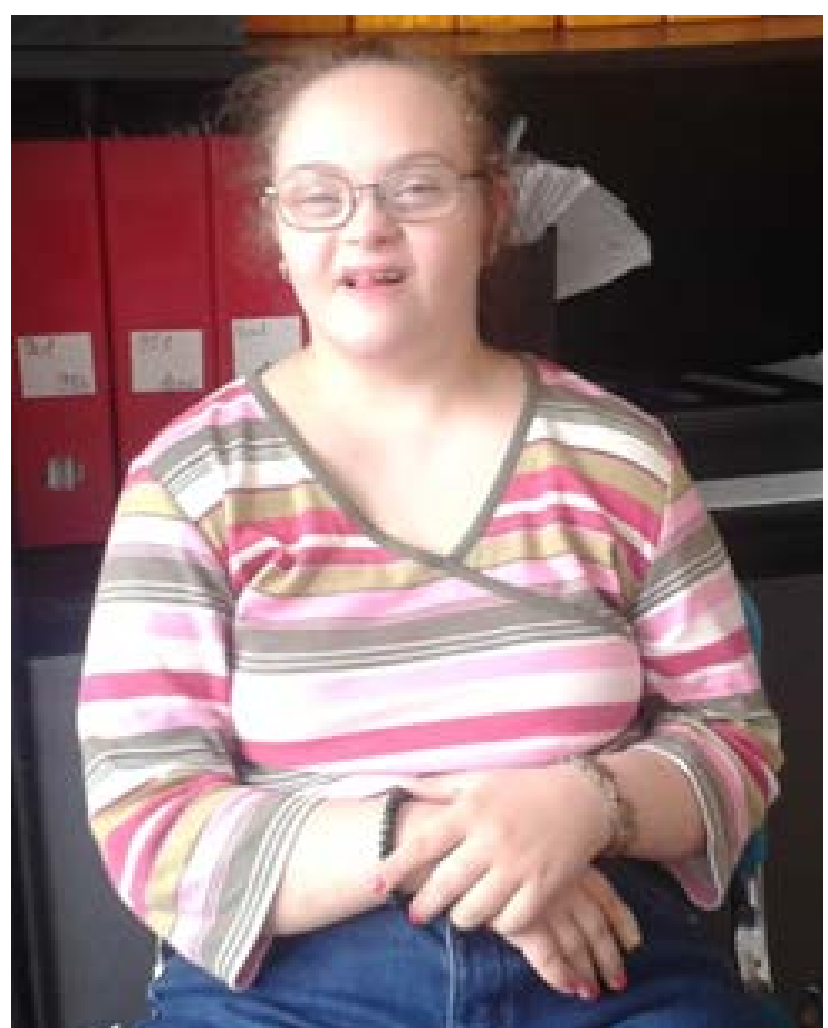

Figure 1-Onset - XI.2014

Biochemistry : $\mathrm{CBC}$ - normal: SGPT $=50 \mathrm{U}$ / L; $\mathrm{SGOT}=34 \mathrm{U} / \mathrm{L}$; HDL-cholesterol = $38.2 \mathrm{mg}$ / $\mathrm{dl} ; \mathrm{ASO}=400 \mathrm{U} / \mathrm{ml}$; HbA1c $=5.5 \%$; EKG: SR- 122 $\mathrm{b} / \mathrm{min}$ without terminal rhythm disturbances; Cardiac ultrasound: without heart defects.

Exophthalmometry (Hertel exophthalmometer) and ocular pressure (tonometer) noticed the absence of the characteristic infiltrative ophthalmopathy disorder (OD -16 mm, OS- 14mm; OD: $15 \mathrm{mmHg}$, OS: $16 \mathrm{mmHg})$.

Abdominal ultrasound: gallbladder - normal shape, slightly hypotonic, fine hypoechoic sludge; left ovary - transonic image of approx. $12 \mathrm{~mm}$ (ovarian functional cyst). Chest x-ray : normal lung, normal cardiac silhouette. (Fig.2). EKG rhythm- revealed sinus tachycardia (Fig. 3). 


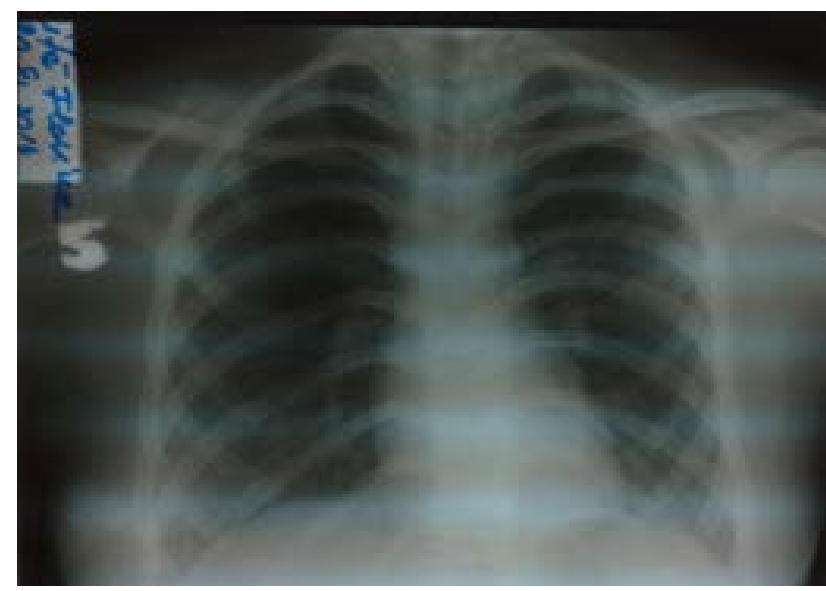

Figure 2: Chest x-ray

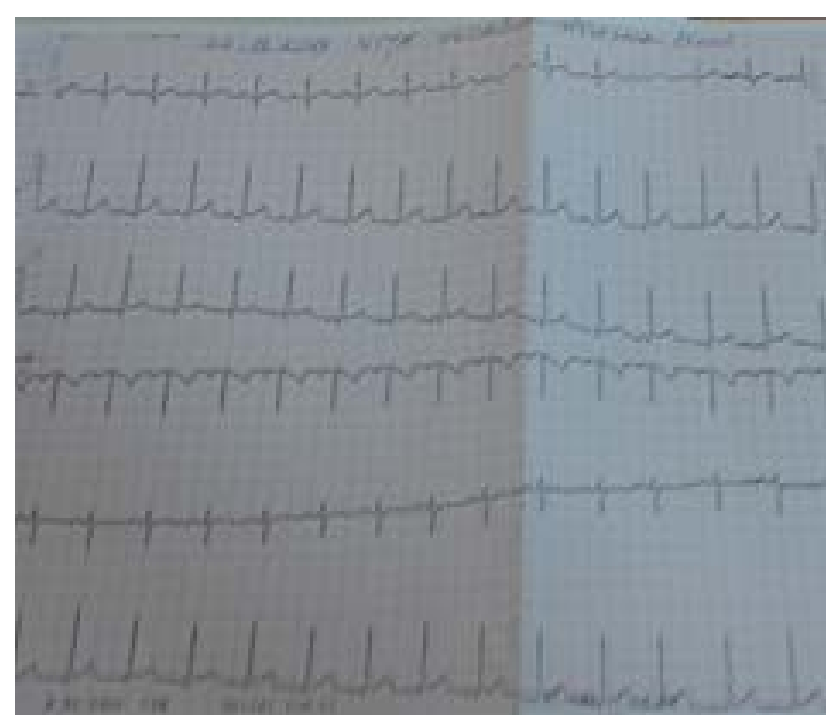

Figure 3: EKG

Thyroid ultrasound : Increased thyroid volume (18 ml-Brunn formula), intense hypoechoic aspect, infiltrative type, heterogeneous echostexture. Intensified intrathyroidal Doppler signal. (Figure 4).

By using the data obtained from history, clinical and paraclinical examinations, the diagnosis was established: Graves' disease, I degree Obesity, Orizontal strabismus, Medium myopia , Luteal insufficiency.

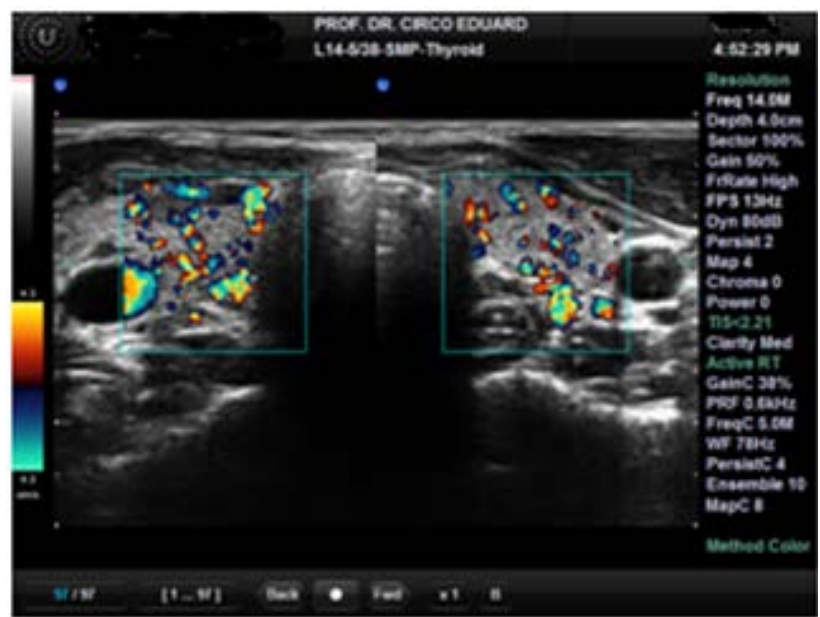

Figure 4: Ultrasouns image - onset of diseasehyperpulsatility of thyroid tissue.

Antithyroid treatment is initiated: Thyrozol of $20 \mathrm{mg}$ /day with good gastric tolerance, without any allergic skin phenomena; as $\beta$-blockers- Propranolol $60 \mathrm{mg} /$ day was initially associated, reduced to $30 \mathrm{mg} /$ day after 5 days. Favourable evolution during hospitalization, discharged with improved general condition after 7 days.

Reassessments (Table I):

I.2015 - Clinical examination - HR $=78 \mathrm{~b} /$ min, diminished agitation and sweating, weight gain aprox. $2 \mathrm{~kg}$; laboratory findings: $\mathrm{TSH}=0.058$ microIU / ml under Thyrozol therapy with $15 \mathrm{mg} /$ day; GPT$32 \mathrm{U} / \mathrm{L}$; It was decided to continue the treatment at the same dose.

II.2015: $\mathrm{TSH}=68.69 \mathrm{microIU} / \mathrm{ml}$; FT4 $=3.8$ $\mathrm{pmol} / \mathrm{L}$; The dose of Thyrozol was reduced to 5 mg / day; Clinical examination: HR- 68 b / min, no sweating and no psychomotor agitation.

IV.2015: TSH - 38.1 microIU / ml; FT4 = $6.05 \mathrm{pmol} / \mathrm{L}$; We initiated the "block and replace" therapy with Thyrozol $5 \mathrm{mg} /$ daily + Euthyrox 50 micrograms/day;

VI.2015: $\mathrm{TSH}=3.2 \mathrm{microIU} / \mathrm{ml} ; \mathrm{FT} 4=16$ pmol / L. Clinical examination: I degree goiter (WHO); HR= $72 \mathrm{~b} / \mathrm{min}$, regularly, no sweating, no palpitations; It was decides to continue the treatment with the same dose for Thyrozol and Euthyrox. Improved ultrasound aspect, decreased thyroid volume $\left(12 \mathrm{~mm}^{3}\right)$ and a significant decreases of Doppler vascular signal (Fig.5). 


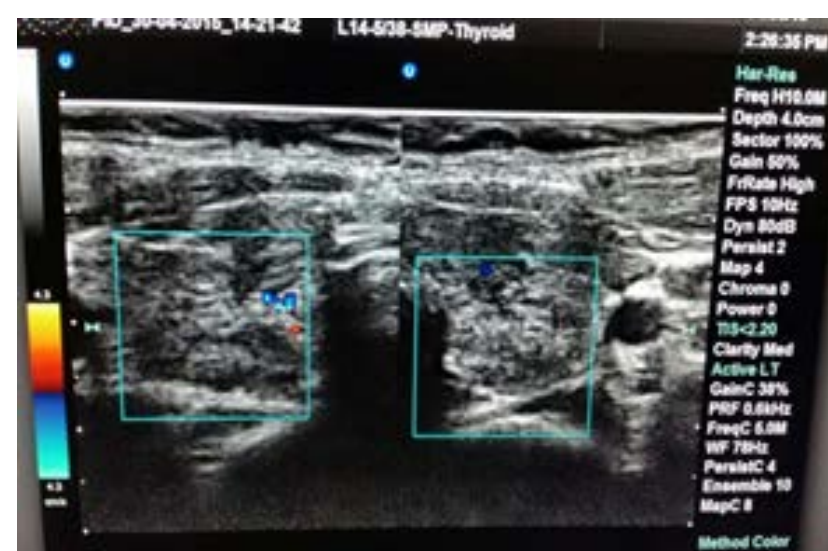

Figure 5: Functional remission.

The patient remains under medical attention for periodic reassessments in ambulatory service.

Table: 1- Phased evolution of serum thyroid hormones and antithyroid medication

\section{Discussions:}

- The study of Gaday-Amo et al.[2] in 2009, on a series of 1832 patients, established an increased incidence of Graves' disease $(6.5 \%)$ in pediatric patients with DS, compared to the general population, which confirm that this kind of pacients present an higher risc to develop an autoimune disorder[8].

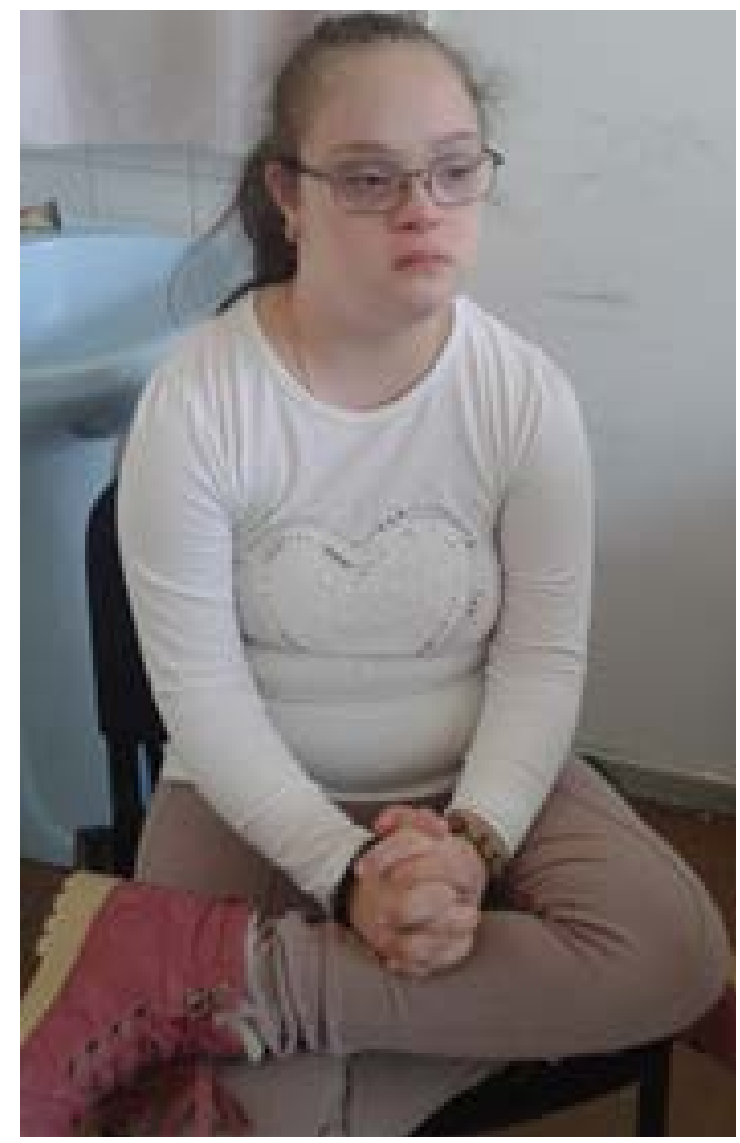

Figure 6: Clinical aspect -remission- VI.2015

- Clinical aspects of Graves disease in DS has several features compared to the general population: young age at onset, lack of predominance of females, low prevalence of ophthalmopathy, low relapse rate after treatment with methimazole and increase remission rates, less severe clinical course, a higher incidence of Hashimoto's thyroiditis in previous medical history, frequently associated with

Table: I- Phased evolution of serum thyroid hormones and antithyroid medication

\begin{tabular}{|l|l|l|l|l|}
\hline Reassesments & TSH, microIU/ml & FT4, pmol/L & THERAPEUTICAL DOSE & \\
\hline I.2015 & 0,058 & 46 & Thyrozol $15 \mathrm{mg} /$ day & Tireotoxicosis \\
\hline III.2015 & 68,69 & 3,8 & Thyrozol is reduced to 5 mg/day; & Iatrogen hypothyroidism \\
\hline IV.2015 & 38,1 & 6,05 & $\begin{array}{l}\text { "block and replace “ therapy is } \\
\text { intitated: Thyrozol 5 mg/day + } \\
\text { Euthyrox 50 microgr; }\end{array}$ & Iatrogen hypothyroidism \\
\hline VI.2015 & 3,2 & 16 & $\begin{array}{l}\text { Continues the treatment in the } \\
\text { same dose }\end{array}$ & Normothyroidism \\
\hline
\end{tabular}


other autoimmune diseases.[2] In the same study no patients with DS needed non-pharmacological therapy (surgery or radioiodine) compared to the control group were non-pharmacological therapy used in $11 \%$ of cases.[2,7]

- Graves disease onset in women in adolescence (puberty) points towards a link between the role of estrogens in the pathogenesis of Graves' disease. Estrogen receptors have been identified in thyroid tissue in $70 \%$ of patients with GD in comparison to $13.3 \%$ in the control group of the study by Wang et al. suggesting the role of estrogen receptor as a promoter factor for GD, remaring that estrogen receptor beta gene polymorphism may be associated with predisposition to Graves's disease.[6,9].

- The presence of anti-TPO-Ab may suggest a chronic autoimmune thyroiditis in patient's history, but the lack of a previous dosage before the onset of symptoms hyperthyroidism doesn't certify this. The association in the family history of thyroid pathology may be an additional factor for sustaining autoimmune aetiology.

- The distinctive characteristic of the case presented impacts on : the symptomatology onset during puberty, mild clinical course, no eye injury, relative fast morphological and functional remission after antithyroid treatment initiation, lack of adverse effects after treatment, the favourable evolution under the "block and replace" therapy.

\section{Conclusions}

- Childhood and adolescence among patients with Down syndrome is often associated with a range of endocrine disorders.

- Monitoring and periodic reassessment of thyroid function is an important aspect in the therapeutic management of these children.

\section{Acknowledgements:}

We thank to the patient for giving us the informed written consent.

$"$ This work received financial support through the project entitled "CERO - Career profile: Romanian Researcher", grant number POSDRU/159/1.5/S/135760, cofinanced by the European Social Fund for Sectoral Operational Programme Human Resources Development 20072013”.

\section{References}

1. Hassold, T.J. \& Jacobs, P.A. (1984). Trisomy in Man . Annual Review of Genetics. (18), 69-97. DOI: 10.1146/annurev.ge.18.120184.000441.

2. De Luca, F., Corrias, A. \& Salerno, M. (2010). Peculiarities of Graves' disease in children and adolescents with Down's syndrome. European Journal of Endocrinology.(162), 591-595. DOI: 10.1530/EJE-09-0751.

3. Popova, G. \& Paterson,W.F. (2008). Hashimoto's thyroiditis in Down's syndrome: clinical presentation and evolution. Hormone Research . (70), 278-284. DOI: 10.1159/000157874.

4. Ivarsson, S.A., Ericsson, U.B. \& Gustafsson J. (1997).The impact of thyroid autoimmunity in children and adolescents with Down syndrome. Acta Paediatrica. (86), 1065-1067. DOI: 10.1111/ j.1651-2227.1997.tb14808.x.

5. Karlsson, B. \& Gustafsson, J. (1998). Thyroid dysfunction in Down's syndrome: relation to age and thyroid autoimmunity, Arch Dis Child (79), 242-245 DOI:10.1136/adc.79.3.242

6. Wang, S. \& Mao,S. (2000). Relationship between estrogen receptor and Graves' disease. Zhonghua Wai Ke Za Zhi. 38(8), 619-621.

7. Le Berre, J.P., Rousseau, C., Dupuy, O.\& Bordier, L. ( 2004). Exciting evolution of auto-immune 
hypothyroidism: occurrence of Graves' disease. Rev Med Interne. (25), 841-843.

8. Roizen, N.J. \& Patterson, D. (2003). Down' s syndrome. The Lancet.(361), 1281-1289. DOI: $\quad$ http://dx.doi.org/10.1016/S01406736(03)12987-X

9. Kisiel, B., Bednarczuk, T. \& Kostrzewa,G. (2008). Polymorphism of the oestrogen receptor beta gene (ESR2) is associated with susceptibility to Graves' disease. Clin Endocrinol (Oxf). 68(3), 429-34. DOI: 10.1111/j.13652265.2007.03060.x. 